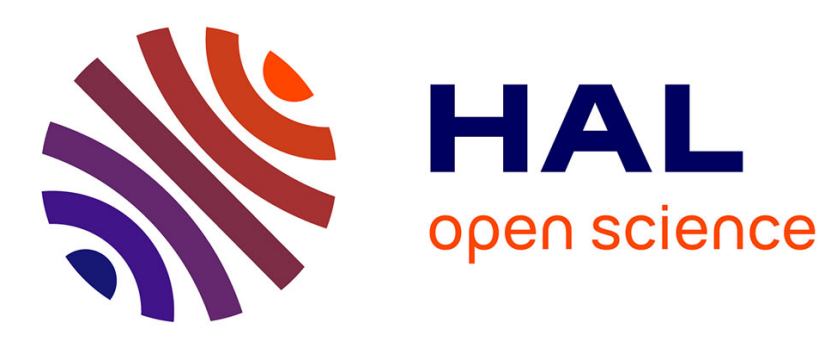

\title{
Saponins from Chenopodium album
}

Catherine Lavaud, Laurence Voutquenne, Philippe Bal, Isabelle Pouny

\section{To cite this version:}

Catherine Lavaud, Laurence Voutquenne, Philippe Bal, Isabelle Pouny. Saponins from Chenopodium album. Fitoterapia, 2000, 71 (3), pp.338-340. 10.1016/S0367-326X(99)00166-5 . hal-01996992

\section{HAL Id: hal-01996992 \\ https://hal.univ-reims.fr/hal-01996992}

Submitted on 6 Dec 2021

HAL is a multi-disciplinary open access archive for the deposit and dissemination of scientific research documents, whether they are published or not. The documents may come from teaching and research institutions in France or abroad, or from public or private research centers.
L'archive ouverte pluridisciplinaire HAL, est destinée au dépôt et à la diffusion de documents scientifiques de niveau recherche, publiés ou non, émanant des établissements d'enseignement et de recherche français ou étrangers, des laboratoires publics ou privés. 


\title{
Saponins from Chenopodium album
}

\author{
Catherine Lavaud ${ }^{\mathrm{a}}$, Laurence Voutquenne ${ }^{\mathrm{a}}$, \\ Philippe $\mathrm{Bal}^{\mathrm{a}}$, Isabelle Pouny ${ }^{\mathrm{b}}$
}

\author{
${ }^{a}$ Laboratoire de Pharmacognosie, UPRESA 6013, Faculté de Pharmacie, 51 rue Cognacq Jay, \\ 51096 Reims cedex, France \\ ${ }^{\mathrm{b}}$ Institut Pierre Fabre, CRSN, 3 rue Arianne, Bureaux de l'Espace, Parc Technologique du Canal, \\ 31527 Ramon ille, France
}

The isolation and spectral data of three saponins from the roots of Chenopodium album L. are reported. One of them is a seco-glycoside analogous to compounds that were previously found in species belonging to Caryophyllales.

Keywords: Chenopodium album; Triterpenoids; Saponins; Seco-glycosides

Plant. Chenopodium album L. subsp. album (Chenopodiaceae) roots were collected in the Champagne region in France in July and August, 1996. It is also known as lamb's quarters and is one of most common agricultural weeds.

Uses and biological activity. Formerly, leaves were consumed as vegetable until replaced by spinach; seeds were ground to a flour and fruits were eaten by poultry 1 . The young leaves are used as a salad for human consumption. Plant possesses diuretic, laxative and sedative properties, leaves are used as poultice 2 . 
Previously isolated classes of constituents. Betalain alkaloids, phenolic acids in fruits, betain and oxalic acid in leaves 3 , oleanolic acid and sitosterol in flowers 4 , furanocoumarins 5 and saponins from the seeds 6 .

New-isolated constituents. 3-O- $\beta$-D-Glucuronopyranosyl oleanolic acid (1) or calenduloside E 7-9 (0.0013\%), 3-O- $\beta$-D-glucuronopyranosyl -28- $O$ - $\beta$-D-glucopyranosyl oleanolic acid (2) or chikusetsusaponin IVa 8-10 (0.0025), 3-O-3'-O(2"-O-glycolyl)-glyoxylyl $\beta$-D-glucuronopyranosyl oleanolic acid (3) 9 (0.003).

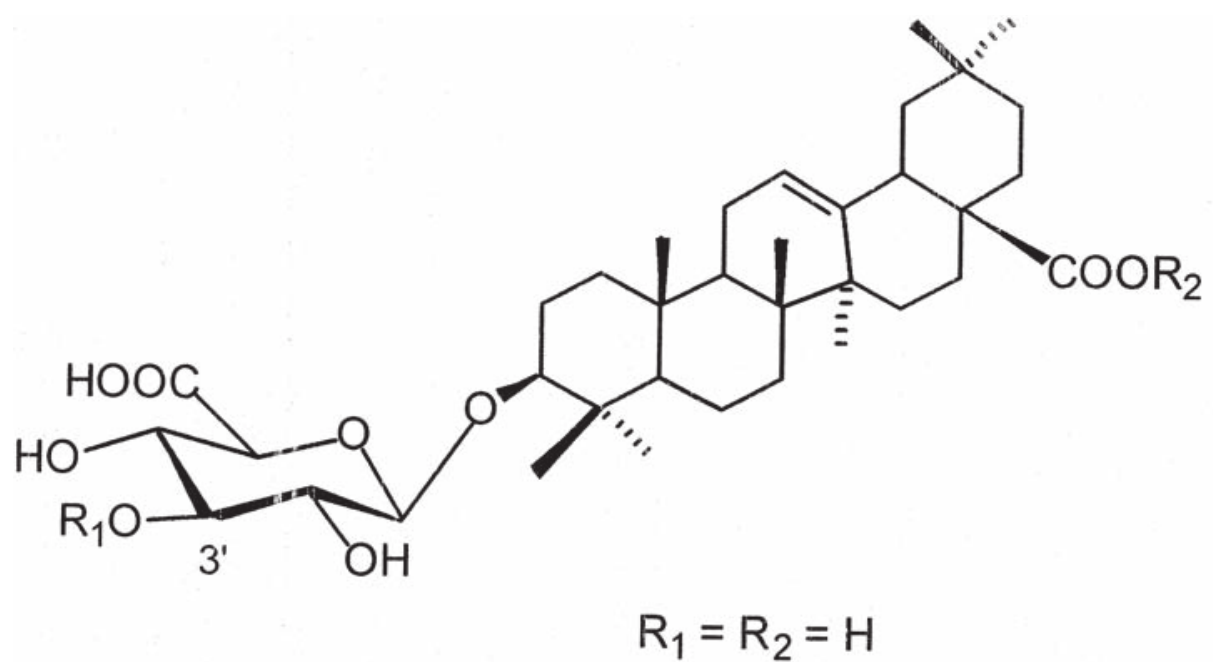

$$
\mathrm{R}_{1}=\mathrm{H}
$$

Calenduloside E (1). ${ }^{1} \mathrm{H}-\mathrm{NMR}\left(500 \mathrm{MHz}, \mathrm{CD}_{3} \mathrm{OD}\right): \delta 0.79(1 \mathrm{H}, \mathrm{brd}, \mathrm{J} 12 \mathrm{~Hz}, \mathrm{H}-5)$, $0.84(3 \mathrm{H}, s, \mathrm{H}-26), 0.86(3 \mathrm{H}, s, \mathrm{H}-24), 0.90(3 \mathrm{H}, s, \mathrm{H}-29), 0.95(6 \mathrm{H}, s, \mathrm{H}-25$ and $\mathrm{H}-30), 1.07(3 \mathrm{H}, s, \mathrm{H}-23), 1.15(3 \mathrm{H}, s, \mathrm{H}-27), 2.88(3 \mathrm{H}, m, \mathrm{H}-18), 3.22(1 \mathrm{H}, d d, J$ 11.7, $4.2 \mathrm{~Hz}, \mathrm{H}-3), 3.27(1 \mathrm{H}, t, J 7.8 \mathrm{~Hz}$, gluA-2), $3.40(3 \mathrm{H}, t, J 9 \mathrm{~Hz}$, gluA-3), 3.45 $(1 \mathrm{H}, t, J 9 \mathrm{~Hz}$, glu-A-4), $3.56(1 \mathrm{H}, d, J 9,5 \mathrm{~Hz}$, gluA-5), $4.35(1 \mathrm{H}, d, J 7.8 \mathrm{~Hz}$, gluA-1), $5.24\left(1 \mathrm{H}, m, W_{1} 27 \mathrm{~Hz}, \mathrm{H}-12\right) ;{ }^{13} \mathrm{C}-\mathrm{NMR}\left(125 \mathrm{MHz}, \mathrm{CD}_{3} \mathrm{OD}\right): \delta 91$ (C-3), 57.1 (C-5), 123 (C-12), 145.5 (C-13) 43.1 (C-18), 28.7 (C-23), 17.2 (C-24), 16.2 (C-25), 18.1 (C-26), 26.7 (C-27), 34 (C-29), 24.4 (C-30), 106.7 (gluA-1), 75.5 (gluA-2), 78 (gluA-3), 73.8 (gluA-4), 75.9 (gluA-5). 
Chikusetsusaponin IVa (2). ${ }^{1} \mathrm{H}-\mathrm{NMR}\left(500 \mathrm{MHz}, \mathrm{CD}_{3} \mathrm{OD}\right): \delta 0.77(1 \mathrm{H}, m, \mathrm{H}-5), 0.81$ $(3 \mathrm{H}, s, \mathrm{H}-26), 0.87$ (3H, s, H-24), 0.92 (3H, s, H-25), $0.93(3 \mathrm{H}, s, \mathrm{H}-29), 0.95$ (3H, $s$, H-30), 1.07 (3H, s, H-23), 1.17 (3H, s, H-27), 2.87 (3H, dd, J 13.7, 3 Hz, H-18), 3.16 $(1 \mathrm{H}, t, J 7.8 \mathrm{~Hz}$, gluA-2), $3.21(1 \mathrm{H}, d d, J 11.7,4.1 \mathrm{~Hz}, \mathrm{H}-3), 3.30(1 \mathrm{H}, m$, glu-2), 3.37 $(1 \mathrm{H}, m$, glu-5), $3.39(3 \mathrm{H}, t, J 9 \mathrm{~Hz}$, gluA-3), $3.43(1 \mathrm{H}, m$, glu-3), $3.45(1 \mathrm{H}, t, J 9 \mathrm{~Hz}$, gluA-4), $3.50(1 \mathrm{H}, m$, glu-4), $3.56(1 \mathrm{H}, d, J 9,7 \mathrm{~Hz}$, gluA-5), $3.70(1 \mathrm{H}, d d, J 11,4.3$ $\mathrm{Hz}$, glu-6), $3.84(1 \mathrm{H}, b r d, J 11.5 \mathrm{~Hz}$, glu-6), $4.35(1 \mathrm{H}, d, J 7.8 \mathrm{~Hz}$, gluA-1), $5.27(1 \mathrm{H}$, $\left.m, W_{1}{ }_{2} 8 \mathrm{~Hz}, \mathrm{H}-12\right), 5.40\left(1 \mathrm{H}, d, J 8.1 \mathrm{~Hz}\right.$, glu-1); ${ }^{13} \mathrm{C}-\mathrm{NMR}\left(125 \mathrm{MHz}, \mathrm{CD}_{3} \mathrm{OD}\right)$ : б 89.2 (C-3), 55.5 (C-5), 122.4 (C-12), 143.3 (C-13), 41.1 (C-18), 27 (C-23), 15.5 (C-24), 14.6 (C-25), 16.3 (C-26), 24.8 (C-27), 176.6 (C-28), 32 (C-29), 22.5 (C-30), 105.3 (gluA-1), 74.1 (gluA-2), 76.6 (gluA-3), 72.3 (gluA-4), 75.1 (gluA-5), 175.6 (gluA-6), 94.2 (glu-1), 72.4 (glu-2), 76.8 (glu-3), 69.6 (glu-4), 77.2 (glu-5), 60.9 (glu-6).

3-O-[3'-O-(2"-O-Glycolyl)-glyoxylyl $\beta$-D-glucuronopyranosyl] oleanolic acid $(\mathbf{3}) .{ }^{1} \mathrm{H}-$ NMR $\left(500 \mathrm{MHz}, \mathrm{CD}_{3} \mathrm{OD}\right): \delta 0.79(1 \mathrm{H}, m, \mathrm{H}-5), 0.85(6 \mathrm{H}, s, \mathrm{H}-24$ and $\mathrm{H}-29), 0.89$ $(3 \mathrm{H}, s, \mathrm{H}-26), 0.96$ (3H, s, H-25), 0.97 (3H, s, H-30), 1.07 (3H, s, H-23), 1.15 (3H, $s$, $\mathrm{H}-27), 2.93(3 \mathrm{H}$, brdd, $J$ 13.5, $3 \mathrm{~Hz}, \mathrm{H}-18), 3.23(1 \mathrm{H}, d d, J 11.7,4.5 \mathrm{~Hz}, \mathrm{H}-3), 3.44$ $(1 \mathrm{H}, m$, gluA-2), $3.58(2 \mathrm{H}, m$, gluA-3 and gluA-4), $3.62(1 \mathrm{H}, m$, gluA-5), $4.05(1 \mathrm{H}$, $\left.d, J 14.8 \mathrm{~Hz}, \mathrm{H}-3^{\prime \prime}\right), 4.35$ (d, $\left.J 14.8 \mathrm{~Hz}, \mathrm{H}-3^{\prime \prime}\right), 4.42$ (1H, $d, J 7.8 \mathrm{~Hz}$, gluA-1), 5.04 $\left(1 \mathrm{H}, s, \mathrm{H}-2^{\prime \prime}\right), 5.22\left(1 \mathrm{H}, m, W_{1} 28 \mathrm{~Hz}, \mathrm{H}-12\right) ;{ }^{13} \mathrm{C}-\mathrm{NMR}\left(125 \mathrm{MHz}, \mathrm{CD}_{3} \mathrm{OD}\right): \delta$ 89.4 (C-3), 55.6 (C-5), 128.4 (C-12), 145.5 (C-13), 42 (C-18), 27.1 (C-23), 15.6 (C-24), 14.5 (C-25), 16.7 (C-26), 25 (C-27), 179.8 (C-28), 32.5 (C-29), 22.9 (C-30), 104.8 (gluA-1), 73.6 (gluA-2), 85.7 (gluA-3), 71.2 (gluA-4), 74 (gluA-5), 178.1 (gluA-6), (C-1" not determined), 101.4 (C-2" ), 67.6 (C-3"), 175.7 (C-4"); ESI-MS (positive mode) $m \quad z: 803.3(\mathrm{M} \quad \mathrm{K})$, (negative mode) $m \quad z \quad 846.8(\mathrm{M} \quad 2 \mathrm{Na} \mathrm{K}$ $3 \mathrm{H}), 786.5(\mathrm{M} \quad \mathrm{Na} \quad \mathrm{H}), 613.5,569.5,455.5$ (aglycone $\mathrm{H}$ ) 100\%, MS-MS (negative mode; $m \quad z$ 763.5) $m \quad z \quad 746.3\left(\mathrm{M}-\mathrm{H}_{2} \mathrm{O}\right), 663.5,631.5$ (M 133) $100 \%$.

\section{References}

1 Mabberley DJ. The plant book. Cambridge: Cambridge University Press, 1993:119.

2 Fournier P. Plantes medicinales. Luxembourg: CME, 1999:tome I, 369.

3 Hegnauer R. Chemotaxonomie der Pflanzen 1964;III:229.

4 Nicholas HJ, Wadkins CL, Hiltibran RC. J Am Chem Soc 1955;77:495.

5 Hegnauer R. Chemotaxonomie der Pflanzen 1989;VIII:229.

6 Stahl E. Z Lebensm-Unters Forsch 1951;93:137.

7 Domon B, Hostettmann K. Helv Chim Acta 1983;66:422.

8 Ridoult CL, Price KR, Parkin G, Dijoux M, Lavaud C. J Agric Food Chem 1994;42:279.

9 Lavaud C, Beauvire S, Massiot G, Le Men-Olivier L, Bourdy G. Phytochemistry 1996;43:189.

10 Lin TD, Kondo N, Shoji J. Chem Pharm Bull 1976;24:253. 